\title{
Caracterización e incidencia de las bibliotecas escolares en el logro educativo en Tamaulipas
}

\author{
Rosa Amelia Domínguez Arteaga*
}

Artículo recibido:

3 de abril de 2019

Artículo aceptado:

16 de agosto de 2019

Artículo de investigación

\section{Resumen}

El objetivo de esta investigación fue examinar las condiciones de las bibliotecas escolares y determinar el grado de correlación lineal entre éstas y el logro educativo en secundarias de Tamaulipas. Para ello se recurre al método estadístico descriptivo. En un primer momento se sistematiza la información disponible a fin de brindar un panorama general de la situación de las bibliotecas, así como el logro académico en el nivel abordado. Posteriormente, se calcula el coeficiente de correlación de Pearson entre las variables disponibilidad de bibliotecas por alumnos y la proporción de alumnos en el nivel IV del área de Lenguaje y Comunicación en secundarias de Tamaulipas del Plan Nacional

\footnotetext{
Departamento de Investigadores Dos, El Colegio de Tamaulipas, Cd. Victoria, México rosa.dominguez@tam.gob.mx INVESTIGACIÓN BIBLIOTECOLÓGICA, vol.34, núm.83, abril/junio, 2020, México, ISSN: 2448-8321 pp. 145-165
} 
para la Evaluación de los Aprendizajes (PLANEA). Por un lado, los resultados indican brechas significativas tanto en la distribución de las bibliotecas como en la proporción porcentual de alumnos en el nivel más alto de la prueba PLANEA entre municipios; por otro, se encontró que existe una relación principalmente negativa entre la disponibilidad de bibliotecas escolares y el logro educativo en las secundarias de los municipios de Tamaulipas.

Palabras clave: Biblioteca Escolar; Logro Educativo; Correlación Biblioteca y Logro Educativo; Tamaulipas

\title{
Characteristics and influence of Library Schools on educational achievement in Tamaulipas \\ Rosa Amelia Domínguez Arteaga
}

\begin{abstract}
The objective of this investigation was to examine the conditions of school libraries and to determine the lineal correlation between them and the educational achievement in secondary schools in Tamaulipas. In order to do so, the method relies upon descriptive statistical procedures. As a first step, a general description of school libraries in the state is presented as well as the educational achievement of the level selected. As a second step, the Pearson correlation coefficient between libraries availability per students and the results of the National Plan for Learning Evaluation (PLANEA) on level IV in the area of Language and Communication was calculated. On the one hand, results show significant gaps between the availability of libraries and the distribution of students on the higher level in the PLANEA test among municipalities. On the other, the correlational results indicate a negative correlation between the availability of school libraries and educational achievement in the state.
\end{abstract}

Keywords: School Library; Educational Achievement; Library Correlation and Educational Achievement; Tamaulipas 


\section{INTRODUCCIÓN}

耳n las directrices de la Federación Internacional de Asociaciones de BiEbliotecarios y Bibliotecas (IFLA) para la biblioteca escolar, se establece que ésta tiene la misión de proporcionar al alumno

información e ideas que son fundamentales para funcionar con éxito en nuestra sociedad de hoy en día, que se basa cada vez más en la información y el conocimiento [...] La biblioteca escolar ayuda a los alumnos a desarrollar destrezas de aprendizaje de carácter vitalicio, así como su imaginación, y les ayuda de esta forma a vivir como ciudadanos responsables. (Unesco, 2002:3)

Por otro lado, en los últimos años se ha establecido la existencia de diferentes variables, elementos, factores y relaciones potenciales que inciden en los procesos de enseñanza, así como en los resultados de aprendizaje de los alumnos, y que pueden ser de tipo institucional, familiar o individual (INNE, 2007). Por tanto, el logro educativo se ha investigado correlacionándolo con otros factores; por ejemplo, con el tema de la inclusión educativa (Sabando, 2016) o con las estrategias y estilos de aprendizaje (Trelles, Alvarado y Montánchez, 2018). En México y en nivel básico, se le ha correlacionado con el ciberacoso (Ortega y González, 2016), así como con factores de eficacia escolar (Eggers, 2016). En el mismo lugar y en escuelas secundarias, se encuentra el estudio que correlaciona la gestión escolar con el logro educativo en centros educativos de Baja California (Gutiérrez, Chaparro y Azpillaga, 2017). En Nuevo León, y más específicamente por área, se han analizado los factores sociodemográficos asociados al rendimiento de los alumnos en Lenguaje y Comunicación y en Matemáticas (González y Rodríguez, 2018).

En ese sentido, otro de los factores que llega a incidir en el logro educativo es la disponibilidad y el uso de la biblioteca escolar. Entre la década de los noventa y a inicios del presente siglo se documentaron varias investigaciones en Estados Unidos que muestran una relación positiva entre las bibliotecas escolares y el logro académico de los alumnos, esto sin importar el nivel socioeconómico del educando (Lance, Hamilton-Pennell y Rodney, 1999; Lance, Wellborn y Hamilton-Pennell, 1993 y 2000; Lance, Rodney y Hamilton-Pennell, 2002; Baughman, 2002; Todd, 2003). Por ejemplo, la participación de la biblioteca representó un aumento de hasta $10.6 \%$ en los resultados en escuelas de Missouri, en Estados Unidos (Miller, Want y Whitacre, 2003), sobre todo en las pruebas de Lenguaje y Letras (Burgin y Brown, 2003; Lance, Rodney y Hamilton-Pennell, 2002). 
Estudios más actuales, como el de Vergara-Lope, Hevia y Rabay (2017), identifican que el acceso a bibliotecas públicas se asocia con las competencias lectoras y de matemáticas. Para el caso de las bibliotecas escolares, Garza y Hernández (2015) observaron una correlación positiva con un coeficiente de Pearson de 0.029 entre el uso de la biblioteca escolar y el rendimiento académico. De forma similar, los estudios de Soria, Fransen y Nackerud (2013 y 2014) encontraron que los estudiantes que obtienen mayores calificaciones son los que leen más, solicitan más libros en préstamo y acceden a los recursos electrónicos. De igual manera, Galicia y Villuendas (2011) encontraron correlaciones estadísticamente significativas entre el rendimiento académico y la frecuencia con la que asisten a la biblioteca, así como con las estrategias de búsqueda de información. Finalmente, Cabral (2008) concluye que la biblioteca, en sus diferentes modalidades, incide en la calidad educativa.

En México, la legislación educativa federal establece que las autoridades educativas centrales y locales deben prestar servicios bibliotecarios que apoyen al sistema educativo, a la innovación educativa y a la investigación científica, tecnológica y humanística (SEP, 2018: 9). Dicha legislación y su réplica en los estados deja ver la importancia de estos espacios para el logro de los objetivos planteados en el sistema educativo nacional, así como en el local (Cárdenas, 2012).

Por ejemplo, y en relación al papel de las entidades al interior del país, la Ley de Promoción a la Lectura y Creación de Libros para el Estado de Tamaulipas $^{1}$ refiere a las bibliotecas escolares como los acervos bibliográficos que la Secretaría de Educación Pública y la Secretaría de Educación de Gobierno del Estado seleccionan, adquieren y distribuyen para su uso durante los procesos de enseñanza y aprendizaje en las aulas y las escuelas públicas de educación básica (POE, 2017b).

Dicha normativa señala, en el artículo 7o. del capítulo tercero, que es menester de la Secretaría de Educación de Gobierno del Estado, en coordinación con los organismos competentes de los Ayuntamientos de la entidad, realizar el Programa Estatal de Fomento a la Lectura y al Libro. Para ello, su fracción VI establece como uno de los medios que la Secretaría de Educación de Tamaulipas (SET) promueva la realización de cursos de capacitación vinculados al trabajo editorial y bibliotecario. Asimismo, en las fracciones IX y X indica que la SET debe garantizar el uso de los acervos de las bibliotecas escolares y cualquier otra medida conducente al fomento de la lectura y del libro (POE, 2017b).

1 Tamaulipas es uno de los 32 estados de la República mexicana, ubicado en el norte del país. Colinda con los estados mexicanos de Nuevo León, por el oeste, y con Veracruz y San Luis Potosí por el sur; hacia el este colinda con el Golfo de México y al norte con el estado estadounidense de Texas. 
Sin embargo, y a pesar de las disposiciones antes mencionadas, las cuales resaltan la importancia de la biblioteca en los procesos educativos, en México estos espacios presentan un desarrollo desigual. Mientras algunas bibliotecas escolares han logrado un reconocimiento por parte de la comunidad escolar, otras apenas inician sus trabajos. Dichas diferencias dependen en gran medida de las condiciones socioeconómicas de los centros escolares, así como de la concepción de directores y maestros sobre la función de la biblioteca en la escuela (SEP y OEI, 2010). Aunado a lo anterior, no existen a la fecha estudios que aborden la situación por entidad federativa en donde se vea reflejada la condición de las bibliotecas y, menos aún, de su impacto entre la comunidad.

Cabe señalar que, con relación a la prueba PLANEA, Tamaulipas se colocó por debajo de la media nacional y ocupó la posición número 11 de menor a mayor puntaje (INEE, 2018b).

Relacionado con Lenguaje y Comunicación para tercero de secundaria, Tamaulipas obtuvo una media de 475 puntos en 2017, con una diferencia de 20 puntos en contra de lo sucedido a escala país, que fue de 495. Además, la entidad retrocedió 28 puntos en comparación con 2015. Sólo el porcentaje de estudiantes en el nivel I mostró diferencia con $40.2 \%$, por encima de la media nacional que fue de $33.8 \%$. Sin embargo, en los niveles II, III y IV, se colocó por debajo de la media nacional, con una diferencia de más de dos puntos porcentuales en los niveles II y III (INEE, 2018b).

En este contexto, el objetivo del presente trabajo es conocer y determinar el grado de relación lineal entre las variables disponibilidad de bibliotecas escolares y el logro educativo en secundarias del estado de Tamaulipas. El alcance del mismo es de tipo descriptivo, exploratorio y cuantitativo.

Se establece como hipótesis de partida que a mayor disponibilidad de bibliotecas escolares por alumno, mayor es el porcentaje de alumnos en el nivel IV de la prueba PLANEA, en particular en el área de Lenguaje y Comunicación, en Tamaulipas. A la fecha, no se encontró un estudio similar que determine la situación de las bibliotecas, y/o la manera como se correlacionan con el logro educativo en dicha entidad y que sirva de base para fines comparativos o de validación de los hallazgos.

Además, esta investigación se justifica en el hecho de que una de las líneas de acción del Plan Estatal de Desarrollo (PED) del gobierno de Tamaulipas es instrumentar un nuevo modelo educativo para mejorar los niveles de aprendizaje y favorecer el tránsito progresivo de los estudiantes hacia un sistema educativo de calidad (POE, 2017a). En ese sentido, los hallazgos de esta indagatoria pueden servir de insumo para el diseño de políticas públicas locales que tomen en cuenta la importancia de la biblioteca escolar, fomentar su uso y con ello alcanzar los objetivos planteados en el sistema local de educación. 
Metodología

\section{Tipo de estudio y método}

El presente estudio se apoyó en el método estadístico descriptivo-correlacional, no experimental, y de corte transversal. Las fuentes de información de donde se obtuvieron los datos para describir de manera general la situación de las bibliotecas, así como el logro educativo, fueron el CEDUTAM (Censo Educativo en Tamaulipas) de 2018, de la Secretaría de Educación de Tamaulipas (SET, 2018), y la prueba PLANEA de 2017 (INNE, 2018a). De dichas fuentes también se determinaron los datos necesarios para la correlación.

Respecto a PLANEA, es una prueba de diagnóstico estandarizada alineada al plan de estudios de educación básica y del Marco Curricular Común de educación superior que evalúa el desempeño de aprendizajes clave del Lenguaje y Comunicación, Matemáticas, y las habilidades socioafectivas de alumnos de educación básica y media superior de carácter público, federal y estatal. Existen tres modalidades de evaluación: Evaluación del Logro referida al Sistema Educativo Nacional (ELSEN), Evaluación del Logro referida a los Centros Escolares (ELCE) y Evaluación Diagnóstica Censal (EDC) (INEE. Unidad, 2015).

En este trabajo se tomó como referencia la prueba ELSEN aplicada los días 14 y 15 de junio de 2017 a 131662 alumnos de tercer grado de secundaria de $3398^{2}$ escuelas en el ciclo escolar 2016-2017. Las asignaturas evaluadas fueron Lenguaje y Comunicación, y Matemáticas (INEE, 2018a). Para este estudio, se delimitó al área de Lenguaje y Comunicación, excluyendo el área de Matemáticas, y se acota a los resultados de nivel sobresaliente (IV) y para alumnos de tercero de secundaria, de manera que se excluyeron los niveles de logro insuficiente (I), apenas indispensable (II) y satisfactorio (III).

Con relación al CEDUTAM (SET, 2018), se trata de un censo aplicado en 2018 a $3410^{3}$ centros educativos, que recaba y sistematiza información fidedigna sobre la situación de los centros escolares en la entidad. El propósito de dicho censo es que sus resultados sirvan de insumo para realizar correlaciones de variables y con ello identificar áreas de oportunidad en investigación educativa. Los datos recabados se relacionan con diferentes variables, tales como liderazgo directivo, funcionamiento institucional, pertinencia de programas sectoriales, población en situación vulnerable y actitudes de los educandos e infraestructura educativa. En esta última variable se les cuestionó a los directores sobre el número de bibliotecas, así como las condiciones de las 
mismas. Como un primer ejercicio exploratorio, sólo se utilizó para este trabajo la variable disponibilidad de biblioteca.

Como nota aclaratoria, cabe señalar que mientras que en PLANEA ${ }^{4}$ los datos son resultado de un muestreo aleatorio, en el caso de CEDUTAM $^{5}$ provienen de un censo en el que participó la totalidad de los centros educativos para el nivel que se aborda en este análisis. Por lo tanto, y para minimizar el sesgo de selección, se llevó a cabo un riguroso tratamiento de las variables, que incluyó, entre otras cuestiones, su operacionalización, normalizar la distribución de las observaciones y la eliminación de valores atípicos. En tal sentido, el conjunto de datos para el que se corrió la prueba de Pearson no se refiere a centros escolares, sino a valores representativos para cada uno de los municipios seleccionados en ambas variables. Esto se describe a continuación.

\section{Detalles de los instrumentos}

Se aplicó el coeficiente o prueba de correlación de Pearson. Éste permite establecer el grado de asociación o dependencia entre dos variables cuantitativas. Para el presente trabajo, las variables a correlacionar y su operacionalización se mencionan a continuación:

- Resultado de logro educativo. Para esta variable se consideró la media del porcentaje de alumnos de tercero de secundaria en el nivel IV de PLANEA en Lenguaje y Comunicación. Se refiere a la proporción de alumnos que cuentan con logro sobresaliente de los aprendizajes clave en dicha prueba.

- Disponibilidad de biblioteca. Se refiere a la proporción de bibliotecas escolares por cada 100 alumnos de secundaria. Es el cociente de la división entre el número total de bibliotecas de secundaria y el total de alumnos de secundaria por municipio. El resultado se multiplicó por 100. Da cuenta de la proporción de alumnos que tienen la posibilidad de acceder y usar los servicios de dichas bibliotecas.

La correlación se valora como significativa en el nivel de $\mathrm{p} \leq .05$. El resultado se expresa en valores de entre -1 y 1 . Cuando el valor $r$ es 0 , entonces se dice que no hay asociación entre las variables. La correlación lineal presupone, a partir de las siguientes hipótesis, que

4 Para la evaluación correspondiente, se contemplaron el número de escuelas y los turnos.

5 Es importante mencionar que en el CEDUTAM se partió del total de los inmuebles, independientemente de los turnos de las escuelas. 
$\mathrm{H}_{0}$ : ambas variables son independientes

$\mathrm{H}_{1}$ : ambas variables están relacionadas

\section{Procedimiento de análisis}

El análisis de los datos se realizó en dos momentos. Primero se hizo uso de la estadística descriptiva a fin de proporcionar un panorama general de las bibliotecas y el logro educativo en Tamaulipas en el nivel secundaria. Para ello, se extrajo del CEDUTAM (SET, 2018) el número de bibliotecas por municipios, y el total de alumnos en el nivel secundaria y por municipio. Paso seguido, se dividieron estos valores entre el número de alumnos matriculados para obtener el porcentaje de alumnos por biblioteca y se multiplicaron por 100. Así también, de la base de datos de PLANEA (INNE, 2018a), se filtró la información para obtener el porcentaje de alumnos en el nivel IV en Lenguaje y Comunicación para cada municipio y así obtener el valor para cada variable. Con ello se pudo obtener una tabla general con los datos a analizar (Tabla 1 en Anexo).

Después, se realizó el cálculo de la correlación de variables para el conjunto de datos con distribución normal y por grupos de municipios. El primer paso consistió en realizar un diagrama de dispersión para observar de manera gráfica la distribución de las variables (Figura 1). De aquí se identificaron valores que afectaban la distribución que deben poseer las mismas (Gorgas-García, Cardiel-López y Zamorano-Calvo, 2011), por lo que se aplicó la prueba de caja en el software SPSS versión 25, tanto para la variable disponibilidad de bibliotecas (Figura 2) como para el caso del porcentaje de alumnos en el nivel IV de PLANEA en Lenguaje y Comunicación (Figura 3).

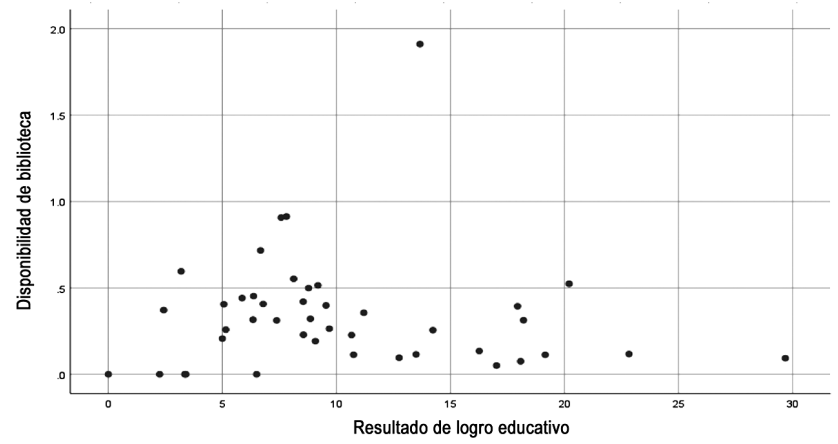

Figura 1. Diagrama de dispersión de variables Fuente: elaboración propia 


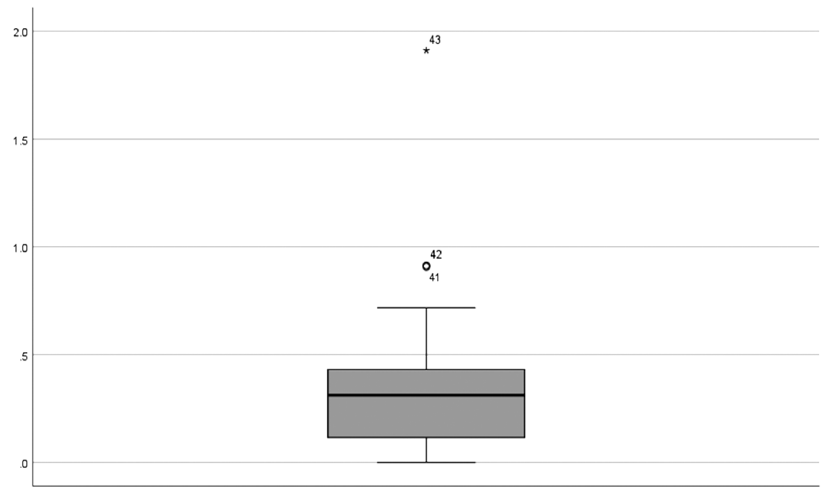

Disponibilidad de biblioteca

Figura 2. Prueba de caja para valores atípicos. Variable Disponibilidad de biblioteca Fuente: elaboración propia

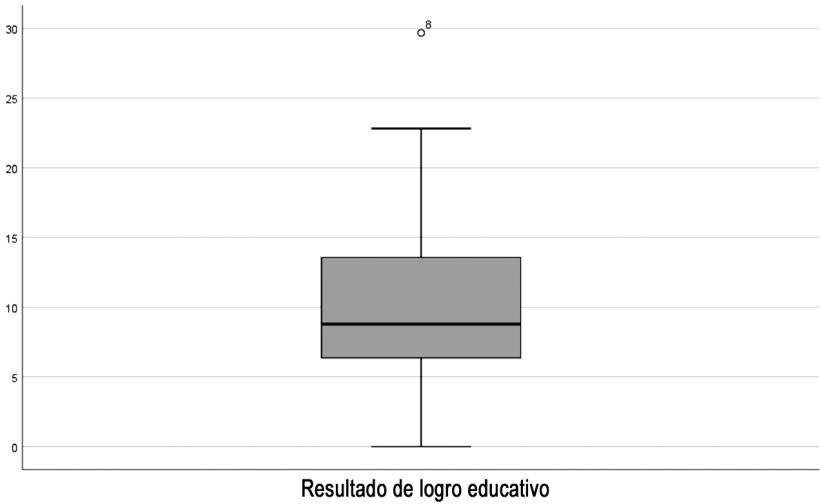

Figura 3. Prueba de caja para valores atípicos. Variable Resultado de logro educativo Fuente: elaboración propia

De dichas pruebas, se detectaron cuatro casos con valores atípicos en total. Para la variable Disponibilidad de biblioteca fueron los casos 41, 42 y 43, y para la variable Porcentaje de alumnos en nivel IV de PLANEA, se ubicó el caso 8 . Aunado a lo anterior, se seleccionaron y excluyeron cinco casos, cuyos municipios contenían valores iguales a 0 en sus variables ( $\mathrm{Ta}$ bla 2 en Anexo). Como resultado, se trabajó con sólo 34 municipios que presentaban una distribución normal.

Por otro lado, para el análisis de la correlación por grupos de municipios, éstos se agruparon en seis bloques según el criterio de disponibilidad de biblioteca. Para ello, primero se calculó el rango que resulta de la sustracción 
del valor más alto y el valor más bajo de las observaciones del conjunto de datos. Para este caso, el resultado fue de 7.118. Posteriormente, se calculó el número de grupos a partir de la aplicación de la raíz cuadrada del número de observaciones y el resultado se redondeó al número más cercano. El resultado fue seis. Finalmente se calculó la longitud de los grupos a partir de la división entre el rango (7.118) y el número de grupos (6) siendo este valor igual a 1.186. Esto permitió establecer los límites inferiores y superiores para cada grupo (Tabla 3 en Anexo).

\section{Presentación y anÁlisis de Resultados}

\section{De la situación de las bibliotecas y el logro educativo en secundarias de Tamaulipas}

La distribución de las bibliotecas en el estado muestra brechas considerables. Mientras que en los municipios de Guerrero, Casas, Mainero, San Nicolás y Cruillas no se reportaron bibliotecas escolares, en el municipio de Tula se contó con 52 unidades. En contraparte, los municipios con mayor número de bibliotecas escolares después de Tula fueron Nuevo Laredo, Matamoros, Reynosa, Altamira y Victoria con 25, 22, 19, 18 y 17 unidades, respectivamente (Tabla 1, Anexo). Además y según la condición de las bibliotecas escolares, $50 \%$ se ubicó en condición "Regular", 37 \% en condición "Bien" y el resto, $13 \%$, en condición "Mal". En números absolutos esto significó 149, 112 y 39 unidades, respectivamente. Destaca el caso de Tula, donde la mayoría de las secundarias fueron calificadas como regulares (Figura 4). En términos generales, la proporción de bibliotecas por alumnos es baja. El municipio con mayor proporción es Tula (1.9), le siguen Abasolo, Jiménez, Méndez y Llera (Figura 5).

Con respecto a PLANEA, la distribución porcentual de alumnos en el nivel IV también indica brechas significativas. Así, mientras que en el municipio de San Nicolás no se ubicaron alumnos en este nivel, Tampico concentró cerca del 30\% de los estudiantes evaluados (Figura 6). Más de $60 \%$ de los municipios del estado (27 unidades) concentraron un porcentaje menor o igual a $10 \%$ de sus alumnos en el nivel IV de PLANEA. Los cinco municipios mejor ubicados según dicha prueba fueron Tampico, Ciudad Madero, Palmillas, Nuevo Laredo y Mier (Figura 6). Al contrario, los cinco municipios con menor porcentaje de alumnos en el nivel IV fueron San Nicolás, Cruillas, Güémez, Llera y Mainero, en ese orden (Figura 6). 


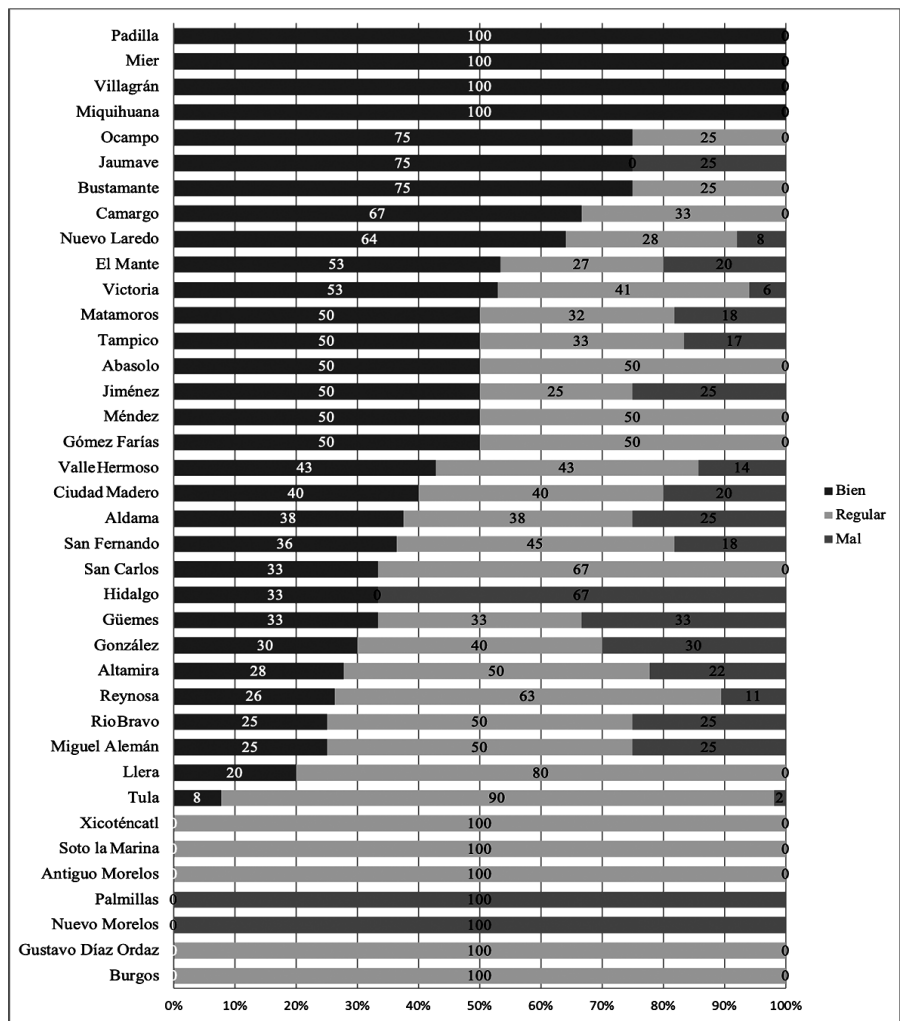

Figura 4. Condición de las bibliotecas en secundarias de Tamaulipas Fuente: elaboración propia, con datos del CEDUTAM (SET, 2018) Nota: se excluyeron los municipios de Casas, Cruillas, Guerrero, Mainero y San Nicolás debido a que no se ubicó respuesta

\section{Resultados de la correlación disponibilidad de bibliotecas y logro educativo en Tamaulipas}

El coeficiente de correlación de Pearson para el conjunto de datos que incluye a los 34 municipios seleccionados con distribución normal arrojó un valor $r$ de -.472. Esto significa que existe una correlación negativa moderada entre la disponibilidad de bibliotecas y el logro educativo. El nivel de significancia es de 0.005 , por lo que se cuenta con evidencia estadística para soportar lo anterior (Tabla 4 en Anexo).

Al correr el estadístico de Pearson para los grupos de municipios seleccionados, se identificó que en todos los casos se obtienen coeficientes 
de correlación distintos de 0 . En cuatro casos el valor es negativo y uno positivo. Las variables están correlacionadas; no obstante, el nivel de significancia es menor a 0.5. Esto significa que la confiabilidad de los resultados es menor a $90 \%$ (Tabla 5 en Anexo).

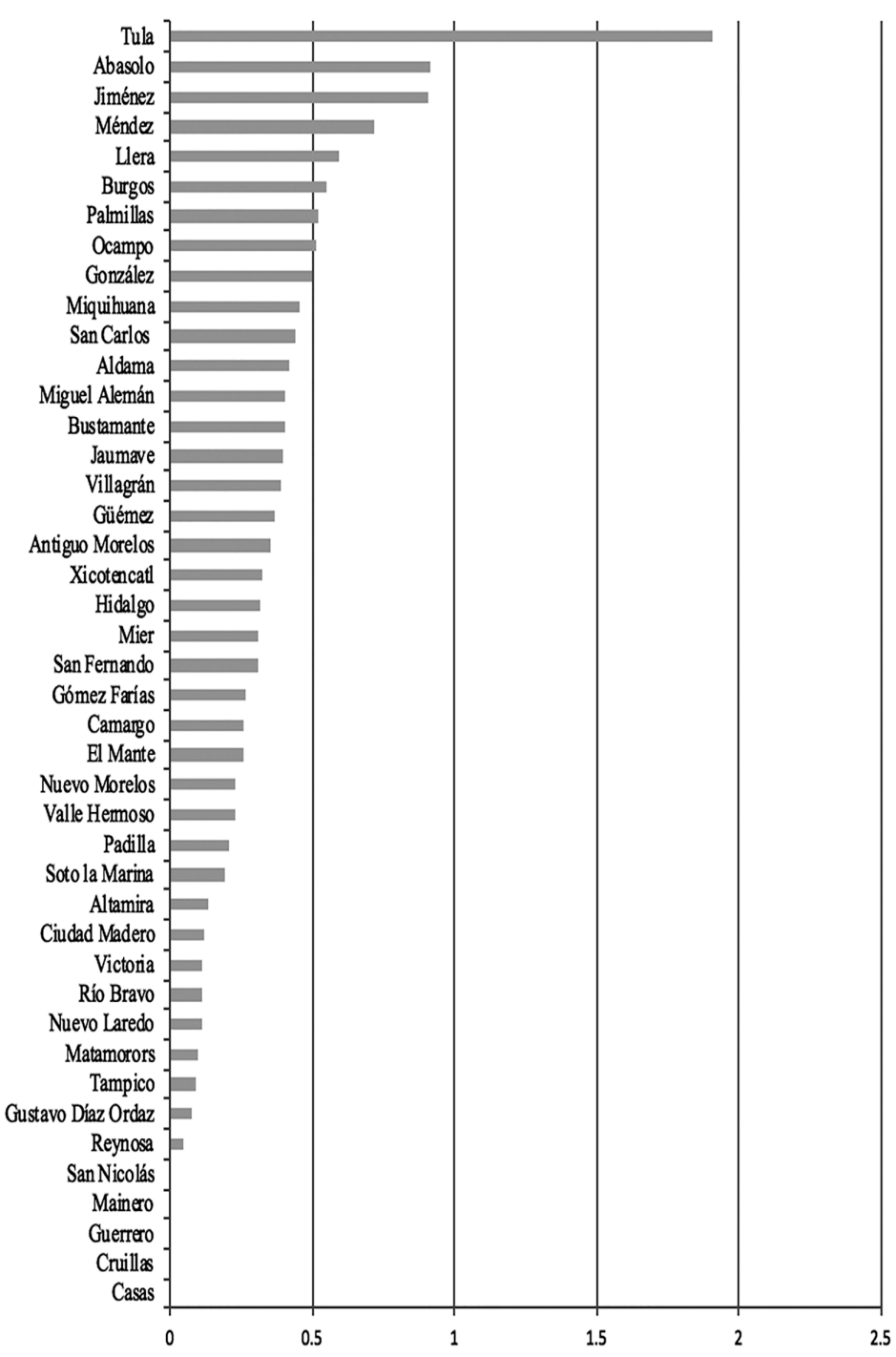

Figura 5. Disponibilidad de bibliotecas por alumno en secundarias de Tamaulipas Fuente: elaboración propia, con datos del CEDUTAM (SET, 2018) 


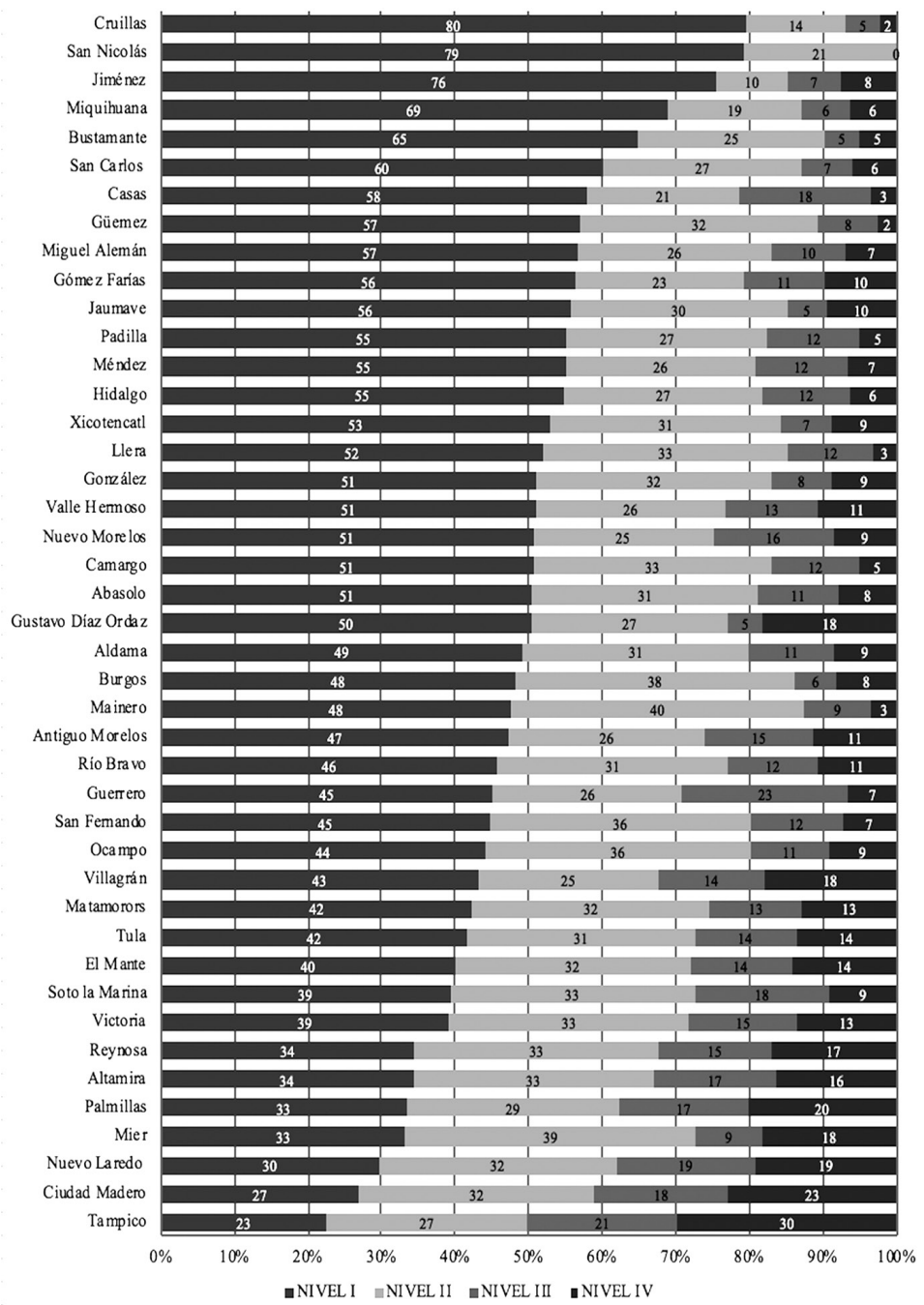

Figura 6. Porcentaje de alumnos de secundaria en prueba PLANEA en Lenguaje y Comunicación de Tamaulipas Fuente: elaboración propia, con datos de INNE (2018a)

\section{Discusión}

En respuesta a los objetivos del presente estudio, los resultados indican que los alumnos de secundaria tienen baja disponibilidad de bibliotecas en el estado. Según los datos reportados por CEDUTAM, se observó que el número de bibliotecas escolares en el nivel analizado es significativamente menor 
con respecto a la población estudiantil en los municipios. Sólo existen en el estado 300 bibliotecas escolares para 176879 alumnos de secundaria. Esto se agrava todavía más debido a que existen municipios que al día de hoy no cuentan con biblioteca en dicho nivel.

Por otro lado, y específicamente para el caso de la correlación por grupos de municipios, los descubrimientos del presente trabajo difieren de otros estudios donde se observa la existencia de una relación positiva y significativa entre la disponibilidad de bibliotecas y logro educativo (Garza y Hernández 2015; Galicia y Villuendas, 2011), y se suma al conjunto de estudios donde se afirma que la biblioteca no incide en los resultados académicos (De Jager, 1997). Si bien la biblioteca es un factor que influye de manera positiva en el logro educativo, la carencia de éstas y una condición regular de las existentes en Tamaulipas explican el porqué de los bajos resultados obtenidos en la PLANEA.

Por lo que se puede decir que Tamaulipas forma parte de la estadística negativa con relación al diagnóstico de las bibliotecas escolares realizado en México y corresponde con los resultados del estudio de la Secretaría de Educación llevado a cabo en 2010 (SEP y OEI, 2010).

Finalmente, destacan situaciones como la del municipio de Palmillas, en donde a pesar de sólo reportar una biblioteca para una población de casi 200 alumnos, se logró un buen nivel en la prueba PLANEA. Estas discrepancias se explican ya que, como bien se estableció, son varios los factores que influyen en el logro educativo, por lo que estos resultados exhortan a analizar, de manera holística, los elementos que inciden de forma positiva en el logro educativo sin dejar de lado el papel de las bibliotecas.

\section{CONCLUSIONES Y RECOMENDACIONES}

Los hallazgos obtenidos sugieren la ausencia de una correlación positiva entre la disponibilidad de bibliotecas y el rendimiento académico de los alumnos de secundaria en Tamaulipas. Una explicación hipotética es que esto se debe a que el número de bibliotecas en todo el estado es insuficiente con respecto del número de alumnos como para incidir de manera clara en los resultados. Cabe señalar que el análisis correlacional no es explicativo, sino que presenta de manera general la ausencia o presencia de asociaciones entre variables. Partiendo de esta premisa, y dados los resultados obtenidos aquí, es altamente recomendable realizar estudios que ahonden en esta relación. Así, es necesario indagar más la correlación que tiene la biblioteca con el rendimiento académico a partir de estudios cuantitativos, pero que además 
contemplen los diferentes indicadores que miden el servicio de la biblioteca escolar. Esto se debe llevar a cabo con muestreos más finos que permitan la aplicación de pruebas estadísticas más rigurosas. Adicionalmente, se sugiere realizar investigación de corte cualitativo que se enfoque, principalmente, en el uso de la biblioteca por parte de los alumnos, así como en el perfil del personal bibliotecario encargado de dichos espacios. En el mismo sentido, convendría determinar las motivaciones de los alumnos para el uso de las bibliotecas escolares.

Con relación al tema de la gestión, se sugiere promover la apertura de bibliotecas escolares en los municipios donde se carece del servicio e implementar acciones de cobertura en los municipios con menos bibliotecas. Si bien es cierto que algunos de estos municipios obtuvieron mejores resultados en la prueba PLANEA, dichos municipios no se encontraron entre los mejores en esta evaluación. Además, y derivado del punto anterior, se recomienda revisar si las características de bibliotecas escolares existentes cumplen con lo estipulado en las directrices al respecto, y toman en cuenta la matrícula de los alumnos para brindar una mejor atención a los usuarios. Se sugiere realizar un censo exclusivamente para bibliotecas escolares, en los que se reflejen, además de las características materiales, el uso de las bibliotecas en las secundarias del estado y evaluar anualmente el rendimiento de las bibliotecas existentes, de acuerdo a las directrices de la Unesco para la biblioteca escolar. Es conveniente establecer mecanismos de seguimiento del uso y aprovechamiento de las bibliotecas escolares. Para ello, se recomienda someter a evaluación -como proceso de mejora continua- al personal encargado de las bibliotecas escolares y evaluar la calidad de los materiales y recursos de las bibliotecas escolares existentes. Para finalizar, un estudio a futuro que se propone es abordar los demás niveles de la prueba PLANEA en Lenguaje y Comunicación, con el objetivo de comparar los resultados que se obtengan al respecto con el nivel analizado en esta entrega.

\section{REFERENCIAS}

Baughman, J. 2002. School Libraries and MCAS Scores. Preliminary Edition. Paper Presented at a Symposium sponsored by the Graduate School of Library and Information Science, Simmons College, Boston, MA, 26 de octubre. http://web.simmons.edu/ baughman/mcas-school-libraries/Baughman\%20Paper.pdf

Burgin, R. y P. Brown. 2003. An Essential Connection: How Quality School Library Media Programs Improve Student Achievement in North Carolina. EUA: RB Software \& Consulting. https://www.lrs.org/documents/impact/NCSchoolStudy.pdf?lrspdfmetric=no

Cabral, B. 2008. "La biblioteca digital y la educación a distancia como entes inseparables para incrementar la calidad de la educación”. Investigación Bibliotecológica 45 (22): 63-78. 
Cárdenas, H. 2012. "La biblioteca y la biblioteca escolar en la legislación educativa mexicana”. Biblioteca Universitaria 2 (15): 147-162. https://www.redalyc.org/articulo.oa?id=28528265004

De Jager, K. 1997. "Library use and academic achievement". South African Journal of Libraries and Information Science 65 (I): 26-30.

Eggers, K. 2016. "Factores de eficacia escolar asociados al aprendizaje de alumnos del sistema de Telesecundaria en México". Tesis doctoral, Facultad de Educación, Universidad Complutense de Madrid, España.

Galicia, J. y E. Villuendas. 2011. "Relación entre hábitos lectores, el uso de la biblioteca y el rendimiento académico en una muestra de estudiantes de psicología”. Revista de la Educación Superior 157 (40): 55-73.

Garza, A. y J. Hernández. 2015. "Factores personales, familiares, escolares, culturales y sociales correlacionados con el rendimiento académico: estudio en alumnos de la Licenciatura en Administración de la UANL”. Vincula Tegica EFAN 1 (1): 2081-2100.

Gorgas-García, J., N. Cardiel-López y J. Zamorano-Calvo. 2011. Estadística Básica para Estudiantes de Ciencias. España: Universidad Complutense de Madrid. Departamento de Astrofísica y Ciencias de la Atmósfera, Facultad de Ciencias Físicas.

Gutiérrez, G., A. Chaparro y V. Azpillaga. 2017. "La organización escolar como variable asociada al logro educativo”. Innovación educativa (México) 74 (17): 41-59.

González, M. y C. Rodríguez. 2018. "Factores sociodemográficos asociados al rendimiento en Lenguaje y Comunicación y en Matemáticas en Nuevo León”. Innovación educativa 76 (18): 105-126.

INNE (Instituto Nacional para la Evaluación de la Educación). 2007. Factores escolares y aprendizaje en México El caso de la educación básica.

https://www.inee.edu.mx/images/stories/documentos_pdf/Publicaciones/Libros_Informes_Capitulos/factores_escolares/factores_escolares_completob.pdf

INEE. Unidad de Evaluación del Sistema Educativo Nacional. 2015. Plan Nacional para la Evaluación de los Aprendizajes (PLANEA). Documentos rectores. http://planea.sep.gob.mx/content/general/docs/2015/PlaneaDocumentoRector.pdf

INEE. 2018a. Evaluaciones de Logro referidas al Sistema Educativo Nacional. Tercer grado de Secundaria, ciclo escolar 2016-2017. Base de Datos PLANEA. https://www.inee.edu.mx/index.php/580-planea

INEE. 2018b. PLANEA. Resultados nacionales 2017. 3 o de secundaria. Lenguaje y Comunicación, Matemáticas.

http://planea.sep.gob.mx/content/general/docs/2017/RESULTADOS_NACIONALES_PLANEA2017.pdf

Lance, K., L. Wellborn y C. Hamilton-Pennell. 1993. The Impact of School Library Media Centers on Academic Achievement. EE UU: Hi Willow Research and Publishing.

Lance, K., C. Hamilton-Pennell y M. Rodney. 1999. Information Empowered: The School Librarian as an Agent of Academic Achievement in Alaska Schools. Alaska, Anchorage: State Library Juneau. Department of Education and Early Development.

Lance, K., L. Wellborn y C. Hamilton-Pennell. 2000. How School Librarians Help Kids Achieve Standards: The Second Colorado Study. Estados Unidos: Hi Willow Research and Publishing. 
Lance, K., M. Rodney y C. Hamilton-Pennell. 2002. How School Librarians Improve Outcomes for Children: The New Mexico Study. Estados Unidos: Hi Willow Research and Publishing.

Miller, J., J. Want y L. Whitacre. 2003. Show-Me Connection: How School Library Media Center Services Impact Student Achievement, 2002-2003. Estados Unidos, MO: Department of elementary and secondary education, Missouri State Library.

Ortega, J. y D. González. 2016. “El ciberacoso y su relación con el rendimiento académico". Innovación Educativa 71 (16): 17-37.

POE (Periódico Oficial del Estado). 2017a. Plan Estatal de Desarrollo 2016-2022, publicado el 31 de marzo de 2017, t. CXLII, Extraordinario número 3. México: Gobierno del estado de Tamaulipas, Poder ejecutivo, Secretaría general. http://po.tamaulipas.gob.mx/wp-content/uploads/2017/03/cxlii-Ext.03310317F.pdf

POE. 2017b. Ley de Promoción a la Lectura y Creación de Libros para el Estado de Tamaulipas, publicado el 27 de agosto de 2013. Última reforma POE No. 14Ext 1512-2017.

https://observatorio.librosmexico.mx/files/2018/leyes-libro/tamaulipas.pdf

Sabando, D. 2016. "Inclusión educativa y rendimiento académico. Relación entre el grado de inclusión y el rendimiento académico en las escuelas públicas de primaria de Cataluña”. Tesis doctoral, Facultad de Educación, Universidad de Barcelona, España.

SEP y OEI (Secretaría de Educación Pública y Organización de Estados Iberoamericanos). 2010. Las bibliotecas escolares en México. Un diagnóstico desde la comunidad escolar. Colección de Estudios. IMéxico: Programa Idea.

SEP. 2018. Ley General de Educación, publicada en el Diario Oficial de la Federación el 13 de julio de 1993. Última reforma publicada DOF 19-01-2018.

https://www.sep.gob.mx/work/models/sep1/Resource/558c2c24-0b12-4676ad90-8ab78086b184/ley_general_educacion.pdf

SET (Secretaría de Educación de Tamaulipas). 2018. Censo Educativo en Tamaulipas (CEDUTAM). Tamaulipas: Subsecretaría de Educación Básica.

Soria, K., J. Fransen y S. Nackerud. 2013. "Library use and undergraduate student outcomes: New evidence for students' retention and academic success". Libraries and the Academy 13 (2): 147-164.

Soria, K., J. Fransen y Sh. Nackerud. 2014. "Stacks, Serials, Search Engines, and Students' Success: First-Year Undergraduate Students' Library Use, Academic Achievement, and Retention”. Journal of Academic Librarianship 40(1): 84-91.

Todd, R. 2003. "Student Learning through Ohio School Libraries: A Summary of the Ohio Research Study". Ohio Educational Library Media Association (OELMA). December 15. http://www.oelma.org/studentlearning.htm

Trelles, H., H. Alvarado y M. Montánchez. 2018. "Estrategias y estilos de aprendizaje y su relación con el rendimiento académico en estudiantes universitarios de Psicología Educativa”. Revista Killkana Sociales 2 (2): 9-16. https://doi.org/10.26871/killkana_social.v2i2.292

Unesco (Organización de las Naciones Unidas para la Educación, la Ciencia y la Cultura). 2002. Directrices de la IFLA/Unesco para la biblioteca escolar.

https://archive.ifla.org/VII/s11/pubs/sguide02-s.pdf 
Vergara-Lope, S., F. Hevia y V. Rabay. 2017. "Evaluación Ciudadana de Competencias Básicas de Lectura y Aritmética y Análisis de Factores Asociados en Yucatán, México”. Revista Iberoamericana de Evaluación Educativa 10 (2): 85-109. https://doi.org/10.15366/riee2017.10.2.005

Para citar este texto:

Domínguez Arteaga, Rosa Amelia. 2020. "Caracterización e incidencia de las bibliotecas escolares en el logro educativo en Tamaulipas". Investigación Bibliotecológica: archivonomía, bibliotecología e información 34 (83): $145-165$.

http://dx.doi.org/10.22201/iibi.24488321xe.2020.83.58100

\section{Anexo}

\begin{tabular}{|c|c|c|c|c|}
\hline Municipio & Bibliotecas & Alumnos & $\begin{array}{l}\text { Disponibilidad } \\
\text { de biblioteca* }\end{array}$ & $\begin{array}{l}\text { Resultado de logro } \\
\text { educativo** }\end{array}$ \\
\hline Abasolo & 6 & 657 & 0.9 & 7.8 \\
\hline Aldama & 8 & 1,904 & 0.4 & 8.5 \\
\hline Altamira & 18 & 13,370 & 0.1 & 16.3 \\
\hline Antiguo Morelos & 2 & 562 & 0.4 & 11.2 \\
\hline Burgos & 1 & 181 & 0.6 & 8.1 \\
\hline Bustamante & 4 & 987 & 0.4 & 5.1 \\
\hline Camargo & 3 & 1,162 & 0.3 & 5.2 \\
\hline Casas & 0 & 161 & 0.0 & 3.4 \\
\hline Ciudad Madero & 10 & 8,527 & 0.1 & 22.8 \\
\hline Cruillas & 0 & 87 & 0.0 & 2.3 \\
\hline El Mante & 15 & 5,873 & 0.3 & 14.2 \\
\hline Gómez Farías & 2 & 758 & 0.3 & 9.7 \\
\hline González & 10 & 2,005 & 0.5 & 8.8 \\
\hline Güémez & 3 & 808 & 0.4 & 2.4 \\
\hline Guerrero & 0 & 126 & 0.0 & 6.5 \\
\hline Gustavo Díaz Ordaz & 1 & 1,332 & 0.1 & 18.1 \\
\hline Hidalgo & 3 & 949 & 0.3 & 6.3 \\
\hline Jaumave & 4 & 1,004 & 0.4 & 9.5 \\
\hline
\end{tabular}




\begin{tabular}{|c|c|c|c|c|}
\hline Jiménez & 4 & 441 & 0.9 & 7.6 \\
\hline Llera & 5 & 839 & 0.6 & 3.2 \\
\hline Mainero & 0 & 162 & 0.0 & 3.4 \\
\hline Matamoros & 22 & 23,012 & 0.1 & 12.7 \\
\hline Méndez & 2 & 279 & 0.7 & 6.7 \\
\hline Mier & 2 & 639 & 0.3 & 18.2 \\
\hline Miguel Alemán & 4 & 983 & 0.4 & 6.8 \\
\hline Miquihuana & 1 & 221 & 0.5 & 6.4 \\
\hline Nuevo Laredo & 25 & 22,178 & 0.1 & 19.2 \\
\hline Nuevo Morelos & 1 & 437 & 0.2 & 8.6 \\
\hline Ocampo & 4 & 777 & 0.5 & 9.2 \\
\hline Padilla & 2 & 968 & 0.2 & 5.0 \\
\hline Palmillas & 1 & 191 & 0.5 & 20.2 \\
\hline Reynosa & 19 & 37,571 & 0.1 & 17.0 \\
\hline Río Bravo & 8 & 7,074 & 0.1 & 10.8 \\
\hline San Carlos & 3 & 680 & 0.4 & 5.9 \\
\hline San Fernando & 11 & 3,528 & 0.3 & 7.4 \\
\hline San Nicolás & 0 & 110 & 0.0 & 0.0 \\
\hline Soto la Marina & 2 & 1,043 & 0.2 & 9.1 \\
\hline Tampico & 12 & 12,907 & 0.1 & 29.7 \\
\hline Tula & 52 & 2,721 & 1.9 & 13.7 \\
\hline Valle Hermoso & 7 & 3,082 & 0.2 & 10.7 \\
\hline Victoria & 17 & 14,772 & 0.1 & 13.5 \\
\hline Villagrán & 1 & 254 & 0.4 & 17.9 \\
\hline Xicotencatl & 5 & 1,557 & 0.3 & 8.9 \\
\hline Totales & 300 & 176,879 & 14.6 & $438^{\star \star \star}$ \\
\hline
\end{tabular}

* Por cada 100 alumnos

${ }^{* *}$ Media del porcentaje de alumnos en Nivel IV de PLANEA

${ }^{* \star \star}$ La suma sin redondear los decimales es 437.6

Tabla 1. Bibliotecas y resultados PLANEA en secundarias de Tamaulipas

Fuente: elaboración propia con datos del CEDUTAM (SET, 2018) y PLANEA, 2017 (INNE, 2018a) 


\begin{tabular}{|c|c|c|c|c|}
\hline Criterio & Región & Municipio & $\begin{array}{l}\text { Disponibilidad } \\
\text { de biblioteca }\end{array}$ & $\begin{array}{c}\text { Resultado } \\
\text { de logro educativo }\end{array}$ \\
\hline \multirow{5}{*}{ 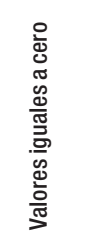 } & Fronteriza & Guerrero & 0 & 7 \\
\hline & Centro & Casas & 0 & 3 \\
\hline & Centro & Mainero & 0 & 3 \\
\hline & Centro & San Nicolás & 0 & 0 \\
\hline & Valle de San Fernando & Cruillas & 0 & 2 \\
\hline \multirow{4}{*}{ 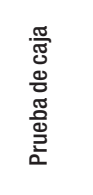 } & Centro & Jiménez & 0.9070 & 7.5750 \\
\hline & Centro & Abasolo & 0.9132 & 7.8087 \\
\hline & Altiplano & Tula & 1.9111 & 13.6609 \\
\hline & Sur & Tampico & 0.0930 & 29.6792 \\
\hline
\end{tabular}

Tabla 2. Municipios excluidos para cálculo del coeficiente de Pearson Fuente: elaboración propia

\begin{tabular}{|c|l|c|c|}
\hline Grupo* & \multicolumn{1}{|c|}{ Municipios } & Límite inferior & Límite superior \\
\hline 1 & $\begin{array}{l}\text { Reynosa, Gustavo Díaz Ordaz, Matamoros, } \\
\text { Nuevo Laredo, Río Bravo, Victoria, Ciudad } \\
\text { Madero }\end{array}$ & 0.051 & 1.237 \\
\hline 2 & $\begin{array}{l}\text { Altamira, Soto la Marina, Padilla, Valle } \\
\text { Hermoso, Nuevo Morelos }\end{array}$ & 1.237 & 2.423 \\
\hline 3 & $\begin{array}{l}\text { El Mante, Camargo, Gómez Farías, San } \\
\text { Fernando, Mier, Hidalgo, Xicoténcatl, } \\
\text { Antiguo Morelos }\end{array}$ & 2.423 & 3.610 \\
\hline 4 & $\begin{array}{l}\text { Güémez, Villagrán, Jaumave, Bustaman- } \\
\text { te, Miguel Alemán, Aldama, San Carlos, } \\
\text { Miquihuana }\end{array}$ & 3.610 & \\
\hline 5 & González, Ocampo, Palmillas, Burgos, Llera & 4.796 & 5.982 \\
\hline
\end{tabular}

*Únicamente el municipio de Méndez se ubicó dentro de los límites inferior (5.982) y superior (7.168) para el sexto grupo, por lo que fue excluido

Tabla 3. Clasificación de municipios según disponibilidad de biblioteca por alumno en secundarias de Tamaulipas

Fuente: elaboración propia 


\begin{tabular}{|l|l|r|r|}
\hline \multicolumn{2}{|c|}{} & $\begin{array}{c}\text { Variable Disponibilidad } \\
\text { de biblioteca }\end{array}$ & $\begin{array}{c}\text { Variable Resultado } \\
\text { de logro educativo }\end{array}$ \\
\hline \multirow{2}{*}{$\begin{array}{l}\text { Variable disponibilidad } \\
\text { de biblioteca }\end{array}$} & Correlación de Pearson & 1 & $-.472^{\star *}$ \\
\cline { 2 - 4 } & Sig. (bilateral) & & 0.005 \\
\cline { 2 - 4 } & $\mathrm{N}$ & 34 & 34 \\
\hline $\begin{array}{l}\text { Variable Resultado de } \\
\text { logro educativo }\end{array}$ & Correlación de Pearson & $-.472^{\star \star}$ & 1 \\
\cline { 2 - 4 } & Sig. (bilateral) & 0.005 & 34 \\
\cline { 2 - 5 } & $\mathrm{N}$ & 34 & \\
\hline
\end{tabular}

**La correlación es significativa en el nivel 0,01 (bilateral)

Tabla 4. Coeficiente de correlación para 34 municipios seleccionados Fuente: elaboración propia

\begin{tabular}{|c|c|c|c|}
\hline $\begin{array}{c}\text { Grupos } \\
\text { de municipios }\end{array}$ & $\begin{array}{c}\text { Coeficiente } \\
\text { de correlación }\end{array}$ & $\begin{array}{c}\text { Nivel } \\
\text { de significancia }\end{array}$ & Dirección y fuerza \\
\hline 1 & -0.047 & 0.920 & $r-$ baja \\
\hline 2 & -0.726 & 0.165 & $r-$ alta \\
\hline 3 & 0.078 & 0.854 & $r+$ baja \\
\hline 4 & -0.089 & 0.834 & $r$-baja \\
\hline 5 & -0.526 & 0.363 & $r$-moderadamente alta \\
\hline
\end{tabular}

*En ninguno de los casos el valor de significancia es menor a 0.5

\section{Tabla 5. Resultados de correlación}

Fuente: elaboración propia 